\title{
CircRNA circPTK2 Might Suppress Cancer Cell Invasion and Migration of Glioblastoma by Inhibiting miR-23a Maturation
}

\section{Wei Chen \\ Ning Wang \\ Minxue Lian}

Department of Neurosurgery, First Affiliated Hospital of Xi'an Jiaotong University, Xi'an City, Shanxi Province, 7I006I, People's Republic of China
Correspondence: Minxue Lian Department of Neurosurgery, First Affiliated Hospital of Xi'an Jiaotong University, No. 277 West Yanta Road, Xi'an City, Shanxi Province, 71006I. People's Republic of China

Email MinxueLianShanxi@163.com
Background: CircRNA circPTK2 plays opposite roles in different cancers, while its role in glioblastoma is unknown. The aim of this study was to explore the involvement of circPTK2 in glioblastoma.

Methods: Expression of circPTK2, mature miR-23a, and premature miR-23a in paired cancer and non-cancer tissues from glioblastoma patients $(n=60)$ was analyzed by RTqPCR. Pearson's correlation coefficient was used to analyze the correlations between gene expressions. The effects of circPTK2 overexpression on miR-23a maturation were analyzed by transfecting circPTK2 expression vector into glioblastoma cells, followed by determining the expression of mature miR-23a and premature miR-23a by RT-qPCR. Transwell assays were carried out to explore the role of circPTK2 and miR-23a in regulating glioblastoma cell invasion and migration.

Results: We found that circPTK2 was downregulated in GBM and was inversely correlated with mature miR-23a, but not premature miR-23a. GBM cells transfected with circPTK2 expression vector showed significantly downregulated mature miR-23a, but not premature miR-23a. Transwell assay analysis showed that circPTK2 overexpression decreased cell invasion and migration, while miR-23a increased cell invasion and migration. Moreover, miR-23a overexpression reversed the inhibitory effects of circPTK2 overexpression on cell behaviors.

Conclusion: CircPTK2 might suppress cancer cell invasion and migration by inhibiting the maturation of miR-23a.

Keywords: glioblastoma, circPTK2, miR-23a, maturation, invasion, migration

\section{Background}

Glioblastoma (GBM) multiforme is a rare type of malignant brain tumor. However, due to its highly aggressive nature, ${ }^{1}$ it has become a common cause of cancer deaths in clinical practice. ${ }^{1}$ GBM is characterized by its dismal outcome and poor prognosis, with a 5-year survival rate of $<5 \%$ and a median survival time of only 12-15 months. ${ }^{2,3}$ Despite the advances in treatment approaches, such as temozolomide-based chemotherapy, surgery, and radiotherapy, treatment of GBM is still technically challenging, mainly due to the development of therapy resistance and infiltrative tumor growth pattern, which could lead to tumor recurrence. ${ }^{1,4,5}$ Therefore, novel therapeutic approaches are needed.

Molecular alterations play critical roles in GBM growth and metastasis. ${ }^{6,7}$ With the increased understanding of molecular pathogenesis of GBM, certain 
molecular pathways, such as $\mathrm{PI} 3 \mathrm{~K} / \mathrm{Akt} / \mathrm{mTOR}$ signaling pathway and Notch pathway, have been proven to be potential targets for the development of novel molecularly targeted GBM therapy, which aims to treat GBM by regulating related gene expression. ${ }^{8-10}$ However, molecular targeted therapy is still under research. Particularly, safe and effective targets remain lacking. Circular RNAs (circRNAs, covalently closed single RNA strands) are emerging critical players in cancer biology. ${ }^{11}$ Rather than coding proteins, circRNAs participate in cancer biology by regulating gene expression at transcriptional or translational levels. ${ }^{12}$ Therefore, circRNAs might be potential targets for targeted therapy. CircRNA circPTK2 plays opposite roles in different cancers. Many studies have certified that circular RNA could function as a tumor promoter or suppressor in the development of various cancers. Recent reports have demonstrated that circPTK2 functions as a tumor promoter in bladder cancer but serves as a tumor suppressor in nonsmall cell lung cancer. ${ }^{13-15}$ Even though the biological function of circPTK2 had been identified in diverse cancers, the specific role of circPTK2 in GBM is unknown. We observed a close correlation between circPTK2 and miR-23a, a critical player in GBM, ${ }^{16}$ in our preliminary microarray dataset (data not shown). We, therefore, analyzed the interactions between circPTK2 and miR-23a in GBM.

\section{Methods}

\section{Patients and Tissue Acquisitions}

This study was approved by the Ethics Committee of First Affiliated Hospital of Xi'an Jiaotong University. At this hospital, we enrolled a total of $60 \mathrm{GBM}$ patients (38 males and 22 females) between June 2017 and June 2020. Their age range was 58 to 72 years, with a mean of $65.4 \pm \pm 6.7$ years. Because other clinical disorders and the application of any therapeutic approaches can affect the expression of certain genes, patients were excluded from any initiated therapy for any clinical disorders within 3 months prior to the admission. Patients complicated with other clinical disorders were also excluded. Recurrent GBM cases and patients with a history of any malignancies were excluded. All the 60 GBM patients were diagnosed for the first time.

Prior to therapy, cancer and paired adjacent (within $3 \mathrm{~cm}$ around tumors) non-cancer tissue samples were collected from the 60 GBM patients using fine-needle aspiration. All samples were confirmed with histopathological analysis and stored in liquid nitrogen prior to the subsequent assays.

\section{GBM Cell Lines and Cell Culture}

Two human GBM cell lines, LN-229 and U-138, from ATCC, USA, were included and cultured in a medium composed of $90 \%$ EMEM (ATCC) and 10\% FBS (ATCC) at $37^{\circ} \mathrm{C}$ in an incubator with $5 \% \mathrm{CO}_{2}$ and $95 \%$ humidity. Both cell lines were cultured to $85 \%$ confluency prior to the subsequent assays.

\section{Cell Transfections}

To overexpress circPTK2 and miR-23a, LN-229 and U-138 cells $\left(10^{8}\right)$ were transfected with circPTK2 expression vector or miR-23a mimic via transient transfections mediated by Lipofectamine 2000 (Invitrogen). The expression vector of circPTK2 was constructed with pcDNA3.1 (Invitrogen) as the backbone. Negative control (NC) miRNA and miR-23a mimic were purchased from Invitrogen. In each experiment, $\mathrm{C}$ (cells without transfection) and NC (empty vector- or NC miRNA-transfected cells) cells were included. Both cell lines were cultured in fresh media for $48 \mathrm{~h}$ after transfection and prior to the subsequent experiments.

\section{Preparations of RNA Samples}

Total RNAs were isolated from paired tissue samples and LN-229 and U-138 cells using RNeasy Mini Kit (QIAGEN) and incubated with DNase I (Invitrogen) at $37{ }^{\circ} \mathrm{C}$ for $100 \mathrm{~min}$ to completely remove genomic DNA. RNA integrity was analyzed electrophoretically on 5\% urea-PAGE gels, and RNA purity was examined using a NanoDrop ${ }^{\mathrm{TM}} 2000$ spectrophotometer by determining OD260/280 ratios.

\section{RT-qPCR Assays}

cDNA samples were prepared through reverse transcriptions (RTs) with RNA samples with satisfactory integrity and an OD260/280 ratio close to 2.0 (pure RNA) as templates. All RTs were completed using a StaRT Reverse Transcription kit (AnyGenes). To determine circPTK2 expression, qPCRs were performed using SYBR Green Master Mix (Bio-Rad) with 18S rRNA as an endogenous control.

To determine the levels of mature miR-23a and premature miR-23a, RTs and qPCRs were performed using All-in-One ${ }^{\mathrm{TM}}$ miRNA qRT-PCR Reagent Kit (Genecopoeia). Prior to analyzing the mature miR-23a 
level, the addition of poly (A) was first performed, and ploy $(\mathrm{T})$ was used as reverse primer. Sequence-specific primers were used to analyze premature miR-23a levels. $\mathrm{Ct}$ values of target genes were normalized to the internal control using the $2^{-\Delta \Delta \mathrm{Ct}}$ method.

\section{Transwell Assay}

To analyze cell invasion and migration, LN-229 and U-138 cells with transfections were subjected to Transwell assays using inserts ( $8 \mu \mathrm{m}$ pore) from Corning. The insert membranes were coated with Corning ${ }^{\circledR}$ Matrigel $^{\mathbb{R}}$ matrix for $12 \mathrm{~h}$ at $37{ }^{\circ} \mathrm{C}$ prior to invasion assays, but not migration assays. To analyze cell migration and invasion, cells in serum-free medium were added into the upper chamber (3000 cells in $0.1 \mathrm{~mL}$ medium per well), and the lower chamber was filled with medium containing $20 \%$ FBS. Cells were cultured at 37 ${ }^{\circ} \mathrm{C}$ for $24 \mathrm{~h}$, and the membranes were collected. After removing cells on the upper surface of the membranes with cotton swabs, cells on the lower surface were stained using $0.5 \%$ crystal violet (Sigma-Aldrich) for $20 \mathrm{~min}$ and counted under a light microscope.

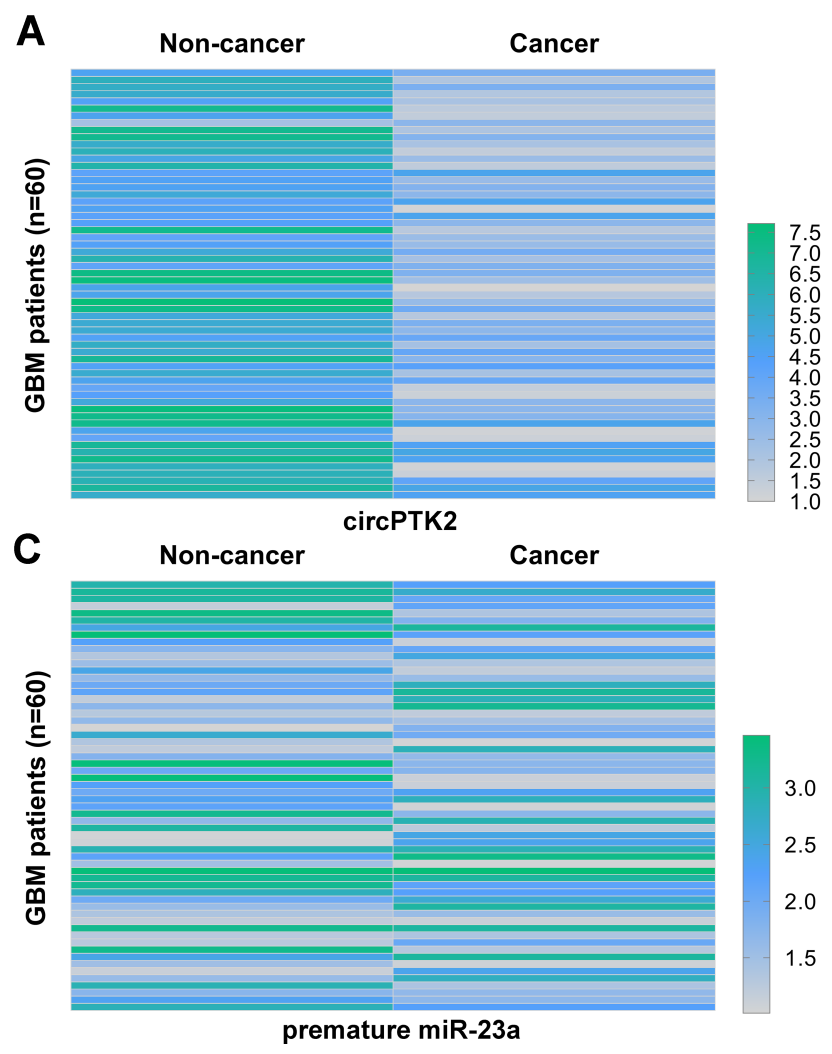

\section{Statistical Analysis}

Data of paired tissue samples were compared by paired $t$-test. Comparisons among multiple independent groups were performed using ANOVA Tukey's test. Pearson's correlation coefficient was applied to analyze correlations. $\mathrm{P}<0.05$ was considered statistically significant.

\section{Results}

\section{GBM Tissues Exhibited Altered Expression of circPTK2 and Mature miR-23a, but Not Premature miR-23a}

Total RNAs were isolated from paired cancer and noncancer tissues of 60 patients who suffered from glioblastoma and used to analyze the expression of circPTK2, mature miR-23a, and premature miR-23a using RTqPCR. HemI 1.0 software was used to plot the heatmaps to represent differential gene expression. Compared to non-cancer tissues, cancer tissues exhibited downregulated circPTK2 (Figure 1A) and upregulated mature miR-23a (Figure 1B). However, no significant difference in

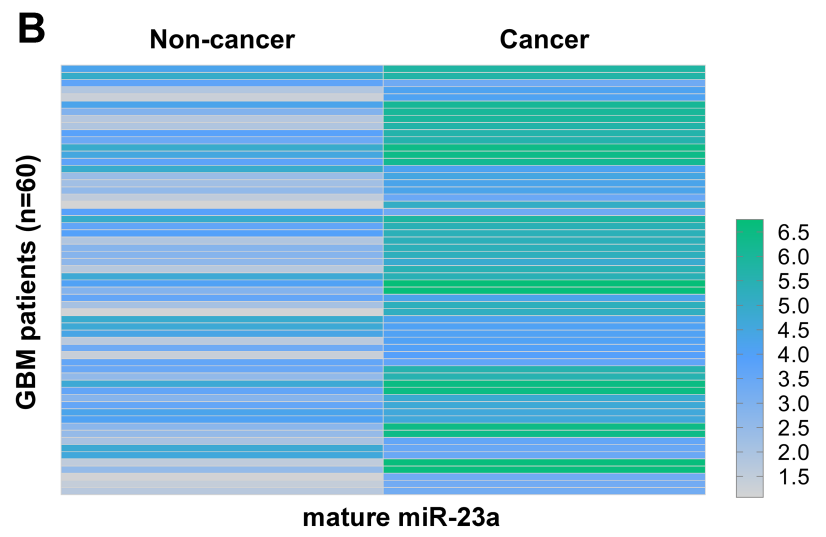

Figure I GBM tissues exhibited altered expression of circPTK2 and mature miR-23a but not premature miR-23a. Total RNAs were isolated from paired cancer and noncancer tissues of 60 GBM patients, and the expression of circPTK2 (A), mature miR-23a (B), and premature miR-23a (C) were analyzed using RT-qPCR. Heml I.0 software was used to plot heatmaps to represent differential gene expression. 

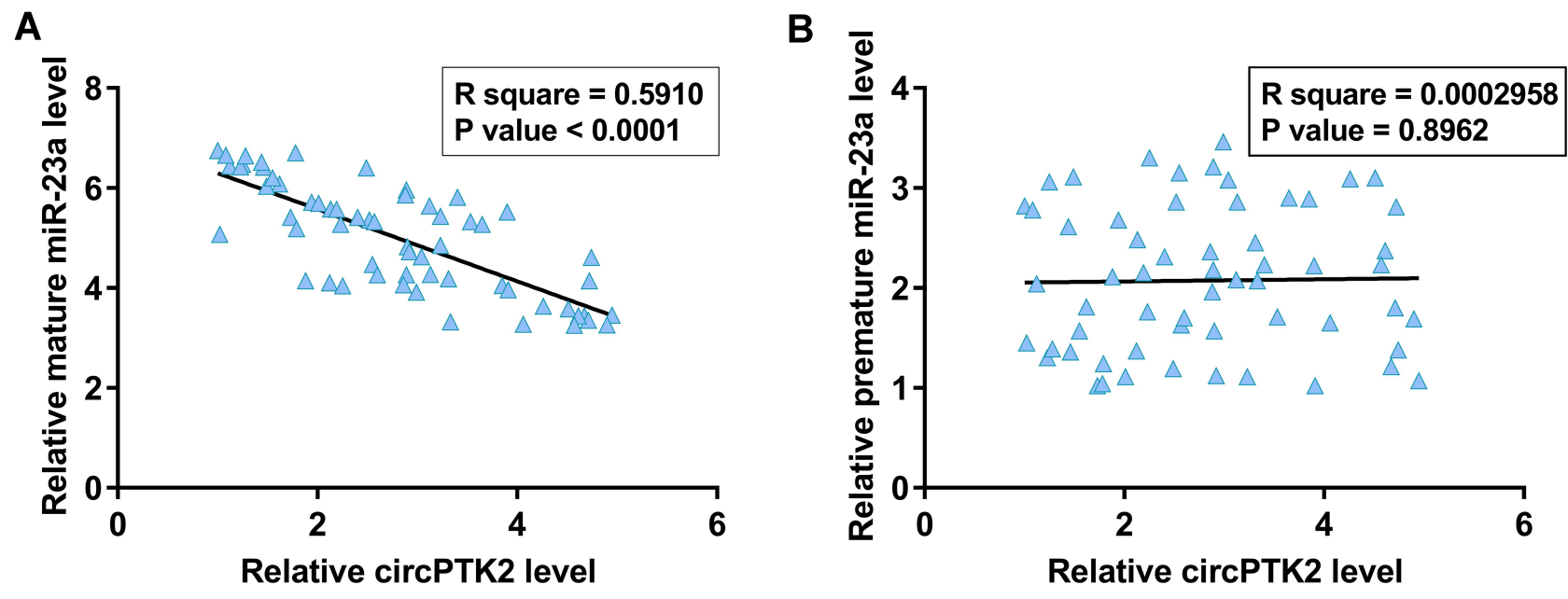

Figure 2 A significant and inverse correlation was observed between circPTK2 and mature miR-23a (A) but not between circPTK2 and premature miR-23a (B).

premature miR-23a expression levels was observed between cancer and non-cancer tissues (Figure 1C).

\section{CircPTK2 and Mature miR-23a Were Inversely Correlated in Glioblastoma Tissues}

Across cancer tissues, the correlations between circPTK2 and mature miR-23a or premature miR-23a were analyzed by Pearson's correlation coefficient. A significant and inverse correlation was observed between circPTK2 and mature miR-23a (Figure 2A) but not between circPTK2 and premature miR-23a (Figure 2B). Therefore, the altered expression of circPTK2 in GBM might affect miR-23a maturation.

\section{MiR-23a Maturation Was Suppressed by circPTK2 Overexpression in Glioblastoma Cell Lines}

The glioblastoma cell lines, LN-229 and U-138, were transfected with circPTK2 expression vector or miR23a mimic, and the overexpression was checked every $24 \mathrm{~h}$ until $96 \mathrm{~h}$. It was observed that circPTK2 and mature miR-23a were significantly overexpressed between $24 \mathrm{~h}$ and $96 \mathrm{~h}$ after transfecting circPTK2 expression vector or miR-23a mimic, respectively (Figure $3 \mathrm{~A}, \mathrm{p}<0.05$ ). LN229 and U-138 cells transfected with circPTK2 expression vector showed significantly downregulated mature miR-23a between $24 \mathrm{~h}$ and $96 \mathrm{~h}$ after transfection (Figure $3 \mathrm{~B}, \mathrm{p}<0.05$ ), while premature miR-23a expression was not affected at each time point (Figure 3C, $\mathrm{p}<0.05$ ). In addition, overexpression of miR-23a mimic failed to significantly alter circPTK2 expression at each time point (Figure 3D). These data suggested that miR23a maturation in glioblastoma cells might be suppressed by circPTK2 overexpression.

\section{CircPTK2 Overexpression Suppressed GBM Cell Invasion and Migration by Inhibiting miR-23a Maturation}

To examine the role of circPTK2 in regulating glioblastoma cell invasion and migration, we performed Transwell assay using LN-229 and U-138 cell lines. Our data showed that circPTK2 overexpression decreased cell invasion and migration, while miR-23a overexpression increased cell invasion (Figure 4A, p<0.05) and migration (Figure 4B, $\mathrm{p}<0.05$ ). Moreover, miR-23a overexpression reversed the inhibitory effects of circPTK2 overexpression on cell behaviors.

\section{Discussion}

This study analyzed the potential involvement of circPTK2 in GBM and explored its interactions with miR-23a. Our data showed that circPTK2 was underexpressed in GBM and could decrease miR-23a maturation to suppress cancer cell invasion and migration.

Interestingly, previous studies have reported the opposite roles of circPTK2 in different types of cancers. ${ }^{13-15}$ In non-small cell lung cancer, circPTK2 is under-expressed, and its overexpression suppresses tumor metastasis and TGF- $\beta$-induced epithelial-mesenchymal transition (EMT) by controlling TIF $1 \gamma .{ }^{13}$ In contrast, circPTK2 is overexpressed in bladder cancer and promotes cancer cell 


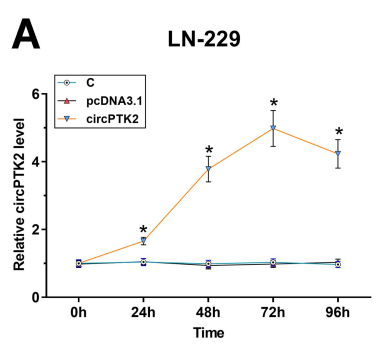

B
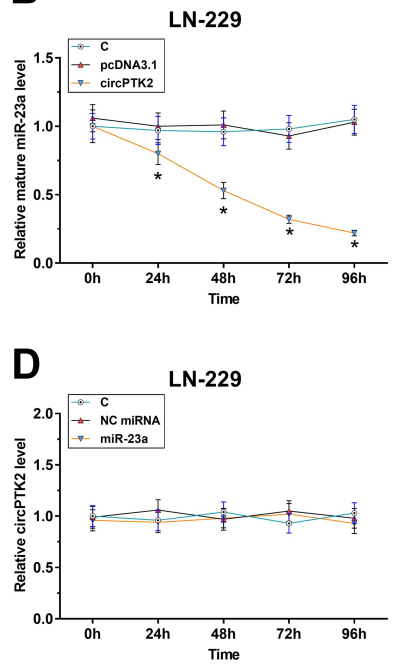

$\mathrm{U}-138$
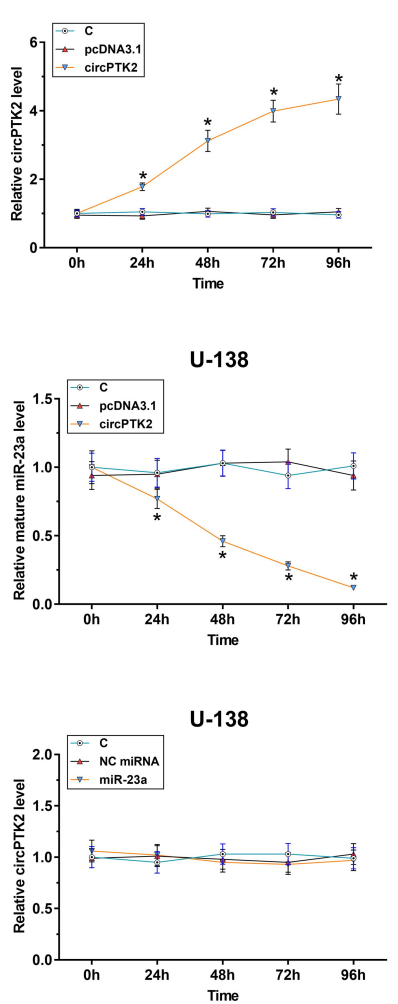

LN-229

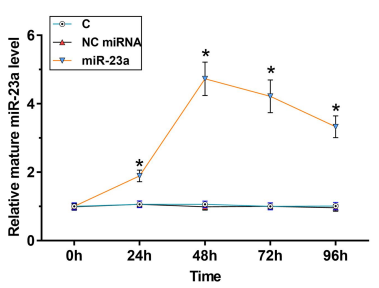

C

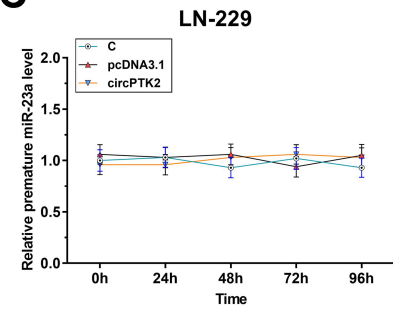

U-138

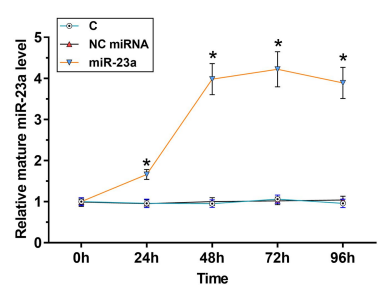

U-138

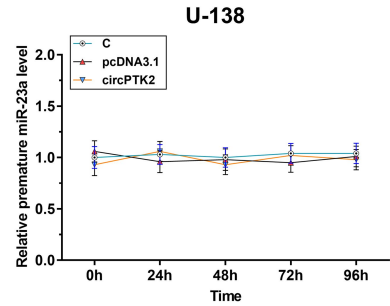

Figure 3 The correlations between circPTK2 and mature miR-23a or premature miR-23a across GBM tissues were analyzed by Pearson's correlation coefficient. LN-229 and U-138 cells were transfected with circPTK2 expression vector or miR-23a mimic, and the overexpression was checked every 24h until 96h (A). The effects of circPTK2 expression vector transfection on mature miR-23a (B) and premature miR-23a $(\mathbf{C})$ and the effects of miR-23a mimic transfection on the expression of circPTK2 (D) at each time point were also analyzed by RT-qPCR. *p<0.05.

migration and proliferation. ${ }^{14}$ Similarly, circPTK2 overexpression was also observed in colorectal cancer, in which circPTK2 binds vimentin to suppress EMT. ${ }^{15}$ Therefore, previously reported functions of circPTK2 cannot be used to speculate its function in other cancers. In this study, we observed the downregulation of circPTK2 in GBM, as well as its inhibitory effects on cancer cell invasion and migration, suggesting the tumor-suppressive roles of circPTK2 in GBM. Therefore, overexpression of circPTK2 might serve as a potential target to suppress GBM metastasis. However, in vivo experiments are needed to verify our conclusions.

MiR-23a also plays different roles in different cancers. ${ }^{16,17}$ For instance, miR-23a is under-expressed in osteosarcoma and targets RUNX2 and CXCL12 to suppress cancer cell invasion and migration. ${ }^{17}$ In contrast, miR-23a is upregulated in GBM and increases cancer cell invasion through HOXD10-regulated glialmesenchymal transition. ${ }^{16}$ Consistently, we also observed the upregulation of mature miR-23a in GBM and its enhancing effects on cancer cell invasion and migration.
However, premature miR-23a was not significantly altered in GBM. Therefore, the increased miR-23a maturation might promote GBM.

Previous studies have shown that circRNAs could interact with miRNAs by serving as their molecular sponges. ${ }^{11,12}$ However, no promising binding site of miR-23a was found in circPTK2. Instead, circPTK2 overexpression in GBM cells suppressed miR-23a maturation. Therefore, we speculate that circPTK2 downregulation in GBM might increase miR-23a maturation, thereby increasing cancer cell invasion and migration. Because the formation of mature miRNAs requires transportation of premature miRNAs from the nucleus to the cytoplasm, circPTK2 might suppress the movement of premature miR-23a. The other possible reason is that the maturation of miRNAs relies heavily on efficient work of the core microprocessor complex, namely, the intranuclear RNase III enzyme Drosha and the double-stranded RNA-binding protein DGCR8. A previous study has demonstrated that circLONP2 indirectly recruit DGCR8/Drosha microprocessor through DDX1 to facilitate the maturation 

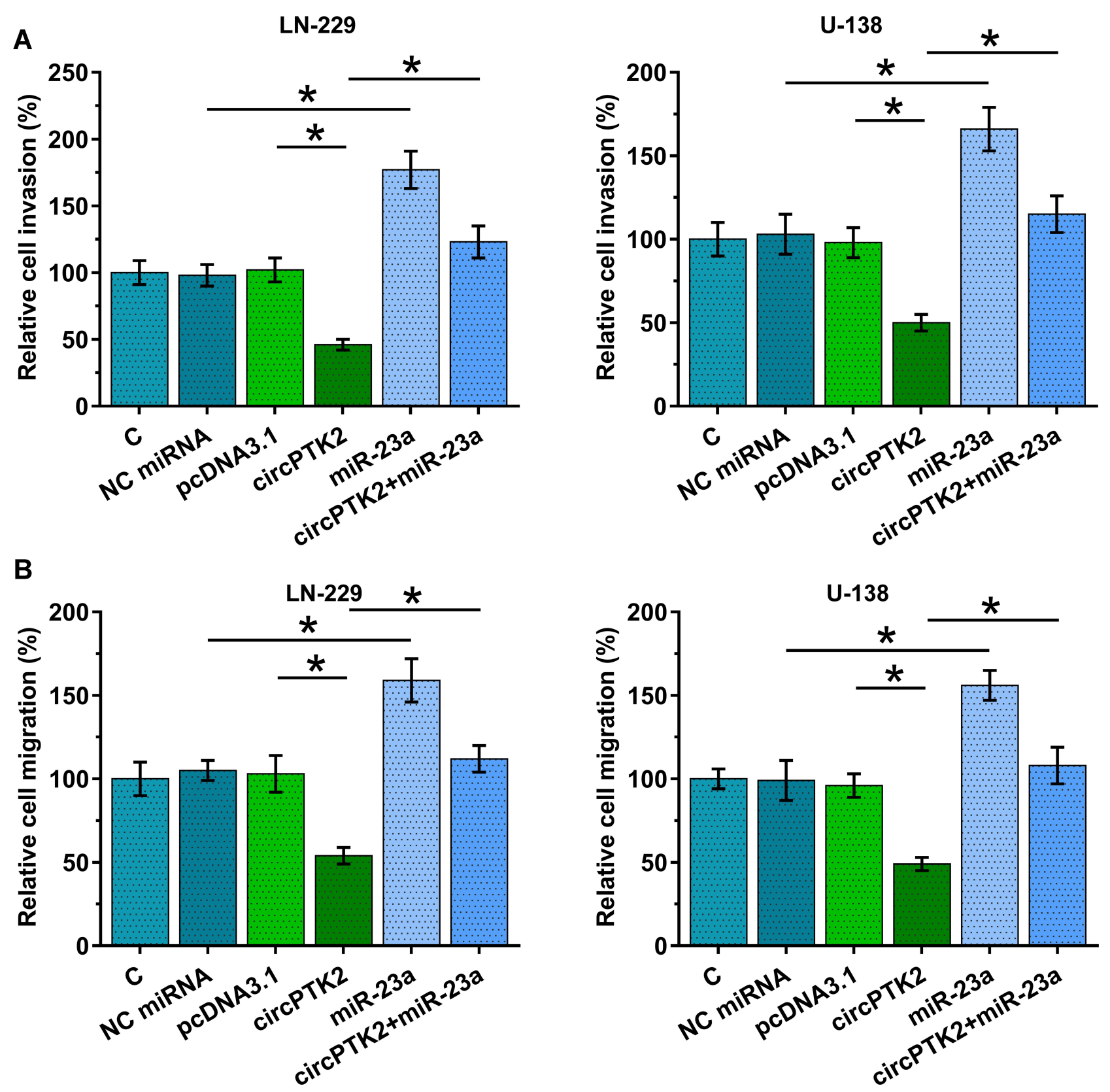

Figure 4 CircPTK2 overexpression suppressed GBM cell invasion and migration via miR-23a. Transwell assay was performed to analyze the role of circPTK2 (A) and miR$23 \mathrm{a}(\mathrm{B})$ in regulating the invasion and migration of $\mathrm{LN}-229$ and $\mathrm{U}-138$ cells. ${ }^{*} \mathrm{p}<0.05$.

processing of pri-miR-17 in regulating colorectal carcinoma invasion and metastasis. ${ }^{18}$ Whether CircPTK2 modulates miRNA biogenesis by inhibiting the miRNA biogenesis pathway needs to be further explored.

\section{Conclusion}

CircPTK2 is under-expressed in glioblastoma and might suppress cancer cell invasion and migration by inhibiting miR-23a maturation.

\section{Availability of Supporting Data}

The data that support the findings of this study are not publicly available due to their containing information that could compromise the privacy of research participants but available on request from the corresponding author: Minxue Lian, Department of Neurosurgery, First Affiliated Hospital of Xi' an Jiaotong University, No. 277 West Yanta Road, Xi'an City, Shaanxi Province, 710061, P. R. China. E-mail:MinxueLianShanxi@163.com. 


\section{Ethical Approval and Consent to Participate}

Informed consent was obtained from all individual participants included in the study. All producers were approved by the Ethics Committee of the First Affiliated Hospital of Xi'an Jiaotong University. Procedures operated in this research were completed in keeping with the standards set out in the Announcement of Helsinki and laboratory guidelines of research in China.

\section{Acknowledgments}

We thank Shaanxi International Cooperation Project for approval.

\section{Author Contributions}

Wei Chen, Minxue Lian: study concepts, literature research, clinical studies, data analysis, experimental studies, manuscript writing and review; Ning Wang: study design, literature research, experimental studies, and manuscript editing. All authors made a significant contribution to the work reported, whether that is in the conception, study design, execution, acquisition of data, analysis and interpretation, or in all these areas; took part in drafting, revising or critically reviewing the article; gave final approval of the version to be published; have agreed on the journal to which the article has been submitted; and agree to be accountable for all aspects of the work.

\section{Funding}

This work was supported by the Shaanxi International Cooperation Project (Grant No. S2017-ZDYF-GJHZMSXM-0115).

\section{Disclosure}

All authors have no conflicts of interest. We declare that we do not have any commercial or associative interest that represents a conflict of interest in connection with the work submitted.

\section{References}

1. Batash R, Asna N, Schaffer P, et al. Glioblastoma multiforme, diagnosis and treatment; recent literature review. Curr Med Chem. 2017;24(27):3002-3009. doi:10.2174/0929867324666170516123206

2. Raj R, Seppä K, Luostarinen T, et al. Disparities in glioblastoma survival by case volume: a nationwide observational study. $J$ Neurooncol. 2020;147(2):361-370. doi:10.1007/s11060-020-03428-5

3. Delgado-López PD, Corrales-García EM. Survival in glioblastoma: a review on the impact of treatment modalities. Clin Transl Oncol. 2016;18(11):1062-1071. doi:10.1007/s12094-016-1497-x

4. Lee CY. Strategies of temozolomide in future glioblastoma treatment. Onco Targets Ther. 2017;10:265-270. doi:10.2147/OTT.S120662

5. Nam JY, De Groot JF. Treatment of glioblastoma. J Oncol Pract. 2017;13(10):629-638. doi:10.1200/JOP.2017.025536

6. Yan D, Hao C, Xiao-feng L, et al. Molecular mechanism of Notch signaling with special emphasis on microRNAs: implications for glioma. J Cell Physiol. 2019;234(1):158-170. doi:10.1002/jcp.26775

7. Wang X, Liu S, Shao Z, et al. Bioinformatic analysis of the potential molecular mechanism of PAK7 expression in glioblastoma. Mol Med Rep. 2020;22(2):1362-1372. doi:10.3892/mmr.2020.11206

8. Festuccia C, Biordi AL, Tombolini V, et al. Targeted molecular therapy in glioblastoma. $J$ Oncol. 2020;2020:5104876. doi:10.1155/ 2020/5104876

9. Li X, Wu C, Chen N, et al. PI3K/Akt/mTOR signaling pathway and targeted therapy for glioblastoma. Oncotarget. 2016;7 (22):33440-33450. doi:10.18632/oncotarget.7961

10. Le Rhun E, Preusser M, Roth P, et al. Molecular targeted therapy of glioblastoma. Cancer Treat Rev. 2019;80:101896. doi:10.1016/j. ctrv.2019.101896

11. Vo JN, Cieslik M, Zhang Y, et al. The landscape of circular RNA in cancer. Cell. 2019;176(4):869-881. e13. doi:10.1016/j.cell.2018.12.021

12. Zhao ZJ, Shen J. Circular RNA participates in the carcinogenesis and the malignant behavior of cancer. RNA Biol. 2017;14(5):514-521. doi:10.1080/15476286.2015.1122162

13. Wang L, Tong X, Zhou Z, et al. Circular RNA hsa_circ_0008305 (circPTK2) inhibits TGF- $\beta$-induced epithelial-mesenchymal transition and metastasis by controlling TIF $1 \gamma$ in non-small cell lung cancer. Mol Cancer. 2018;17(1):140. doi:10.1186/s12943-018-0889-7

14. Xu ZQ, Yang MG, Liu HJ, et al. Circular RNA hsa circ 0003221 (circPTK2) promotes the proliferation and migration of bladder cance cells. J Cell Biochem. 2018;119(4):3317-3325. doi:10.1002/jcb.26492

15. Yang H, Li X, Meng Q, et al. CircPTK2 (hsa_circ_0005273) as a novel therapeutic target for metastatic colorectal cancer. Mol Cancer. 2020;19(1):13. doi:10.1186/s12943-020-1139-3

16. Yachi K, Tsuda M, Kohsaka S, et al. miR-23a promotes invasion of glioblastoma via HOXD10-regulated glial-mesenchymal transition. Signal Transduct Target Ther. 2018;3:33. doi:10.1038/s41392-0180033-6

17. He Y, Meng C, Shao Z, et al. MiR-23a functions as a tumor suppressor in osteosarcoma. Cell Physiol Biochem. 2014;34(5):1485-1496. doi: $10.1159 / 000366353$

18. Han K, Wang F, Cao C, et al. CircLONP2 enhances colorectal carcinoma invasion and metastasis through modulating the maturation and exosomal dissemination of microRNA-17. Mol Cancer 2020;19(1):60. doi:10.1186/s12943-020-01184-8 


\section{Publish your work in this journal}

Neuropsychiatric Disease and Treatment is an international, peerreviewed journal of clinical therapeutics and pharmacology focusing on concise rapid reporting of clinical or pre-clinical studies on a range of neuropsychiatric and neurological disorders. This journal is indexed on PubMed Central, the 'PsycINFO' database and CAS, and is the official journal of The International Neuropsychiatric Association (INA). The manuscript management system is completely online and includes a very quick and fair peer-review system, which is all easy to use. Visit http://www.dovepress.com/testimonials.php to read real quotes from published authors.

Submit your manuscript here: https://www.dovepress.com/neuropsychiatric-disease-and-treatment-journal 